\title{
Challenges facing holographic models of QCD
}

\author{
Thomas D. Cohen* \\ Department of Physics, University of Maryland, College Park, MD 20742-4111
}

\begin{abstract}
This paper, written in memory of Manoj K. Banerjee, takes a critical look at holographic models of QCD focusing on "practical" models in which the five-dimensional theory is treated classically. A number of theoretical and phenomenological challenges to the approach are discussed.
\end{abstract}

Manoj K. Banerjee served as a mentor to me during the early stages of my career; he had a profound influence on my scientific development. Without exaggeration, I can say that I owe my scientific career to him. Thus, I am deeply honored to have an opportunity to write a paper in this issue of the Indian Journal of Physics dedicated to his memory.

I first got to know Manoj in 1985. I had arrived at the University of Maryland as a fresh Ph.D. having done a thesis in nuclear structure physics related to the then trendy Interacting Boson Model. I was looking to expand my horizons. The nuclear theory group at Maryland has an admirable tradition: postdocs are not formally assigned to a given faculty member but can work with whomever they please. I naturally gravitated towards Manoj. In the first place he was doing very interesting science: soliton models of the nucleon[1]. Perhaps equally attractive was the sheer intellectual excitement of his research effort and the obvious and infectious joy and enthusiasm with which he did science.

Manoj's enthusiasm could lead to some very intense scientific discussions. I recall an incident which characterizes the intensity of those days. A scientist was visiting our group to give a seminar on a competing model of the nucleon. Before his talk our visitor, along with me, and Wojeich Broniowski (who at that time was Manoj's Ph.D. student) met in Manoj's office to discuss this model. The discussion became rather heated. Indeed, it became so heated that an undergraduate attending office hours in Wally van Orden's office (a couple of offices away) asked, "Is everything OK in there? It sounds like they are having a fight." It is worth noting though that while Manoj was often heated in his discussion of science, he was always free of malice. Rather, the intensity was driven by a deep need to get to the core of things along with an incisive mind and critical scientific judgment. This critical scientific judgment was omnipresent in Manoj.

One aspect of this which I always appreciated was his extreme reluctance to accept arguments based on appeals to authority. He was also very suspicious of ideas which seemed to be trendy. He also had a rather healthy skepticism toward approaches which where driven by elegant mathematics rather than rooted in the physics. In this context, it is interesting to contemplate what his reaction would have been to the recent emergence of so-called

*Electronic address: cohen@physics.umd.edu "holographic models of QCD", an approach which sometimes goes under the name of $\mathrm{AdS} / \mathrm{QCD}[\underline{3}, 4,4,5,6,6,7,6$, 9, 10, 11, 12, 13, 14, 15, 16, 17, 18, 19]. This approach is based on the hope that some truly spectacular results in mathematical physics arising from issues in string theory might be somewhat modified and then applied directly to QCD. Certainly the approach is trendy and does seem driven by the elegant mathematics. Moreover, one might sense at least an implied appeal to authority as the underlying ideas are taken from Maldecena's remarkable insight relating conformal field theories in four dimensions to anti-deSitter space in five dimensions - the so-called AdS/CFT duality [22, 23, 24]. My suspicion is that Manoj would have a number of deep questions about the entire enterprise of holographic models of QCD.

It seems to me that it is appropriate in this volume dedicated to the memory of Manoj Banerjee to write a paper which celebrates his spirit of critically probing the models in the field. This paper will take a critical look at a few aspects of what has been called the bottom-up approach to holographic QCD. It is not intended as a review of the literature - which in any event is growing rapidly. Rather it is intended to raise a number of questions about the foundations of the approach which will need to be addressed before it can be considered as a viable approach to hadronic physics. It should be noted that none of the issues discussed in this paper are particularly subtle and all should be known at some level to workers in the field. The purpose of this paper is to emphasize at least some of the challenges facing these models and to stress the need for the community to deal with them.

Before proceeding with this discussion, a few words of caution are probably in order. I should noted that Manoj was much more sensible than me. While in person he was always very outspoken about his intellectual concerns about the foundations of various models on the market, he had the good sense not to write articles cataloging them. This was sensible for a number of reasons. One key reason is simply that on occasion concerns about the foundations of a model are not so much wrong as simply off base. This can happen if the aims of a given model are rather modest. Then, it may happen that while the objections may be trenchant, they are only so if the model is taken to apply more widely than is intended. For example, Manoj always had a great skepticism about the Skyrme model[2]. Given what I wrote before, this is hardly surprising: it was trendy, driven as much by elegant mathematics as by physics and often supported 
by appeals to Witten's authority. Of course the Skyrme model was often abused: it was used where it ought not to have been - and Manoj was always quick to spot this. However, I always had the sense that he never fully appreciated that while the model is quite limited - it could only be sensibly used for a narrow class of problems within this limited domain the model makes a good bit of sense, at least as an illustrative model. It is quite likely that a similar situation will arise for the models here. That is, if the models are viewed as having a sufficiently modest regime of applicability, some of the concerns outlined in this paper may not apply. There is a second obvious need for caution. The state of the art in the field is in flux; there is no generally accepted scheme for how one should implement models of holographic QCD. Thus, even if the issues discussed in this paper do invalidate some aspects of the various models presently on the market, it remains quite possible that new variants might be constructed which will evade them. With these words of caution, let us begin our discussion.

The models are holographic in the following sense: it is assumed that QCD (or some other theory which in some sense approximates QCD) lives in a four-dimensional world which is the boundary of a higher-dimensional curved space (typically five dimensional) on which there exists a dual theory with the property that correlation functions obtained by varying sources constrained to the four-dimensional boundary of the dual theory reproduces the correlation functions of QCD (or the theory which approximates QCD). Since all physical information about a theory is effectively contained in its correlation functions one can thus learn properties of QCD by solving its higher-dimensional dual. The models are called "holographic" since information contained in a lower dimension (in this case the boundary theory) allows one to see what is going on in a higher dimension in much the same way that the information in a two-dimensional hologram allows one to see three-dimensional images.

Now, as stated, this construction is generically of little use for studying QCD: if one is replacing one quantum field theory (QCD) that you cannot solve by a higherdimensional quantum field theory which you also cannot solve, you have made no progress. On the other hand, suppose that there is a formal limit for which the higher-dimensional theory becomes weakly coupled and, hence, classical while the four-dimensional theory remains a strongly coupled quantum theory. Then, to the extent one is near this limit, one can solve the higherdimensional theory classically to learn about the quantum physics of the lower-dimensional theory. All models of the bottom-up holographic QCD are based either explicitly or implicitly on the assumption that there exists a five-dimension dual QCD and for that reason the theory is near such a limit allowing a classical treatment of the five-dimensional theory. I will refer to such a model as "practical" since if this condition is false then the fivedimensional theories are not likely to be tractable in practice.
This raises obvious questions: Does a practical holographic dual for QCD exist? If so, what limit of QCD makes the dual theory classical? If a practical holographic dual does not exist, to what extent can practical holographic models mimic key aspects of QCD?

The hope that such a practical theory exists is based on the conjectured dualities between some types of gauge theories and higher-dimensional gravity theories - most notably the AdS/CFT correspondence [22, 23, 24]. In this correspondence, a four-dimension conformal field theory, such as $\mathcal{N}=4 S U\left(N_{c}\right)$ super Yang-Mills theory (in the large $N_{c}$ limit), is dual to a type IIB string theory on $A d S_{5} \times \mathbf{S}^{5} ; A d S_{5}$ is $5 \mathrm{D}$ Anti-deSitter space, and $\mathbf{S}^{5}$ is the 5 -sphere. The remarkable thing is then when the 't Hooft coupling of the conformal field theory is large, the $A d S_{5} \times \mathbf{S}^{5}$ physics is described by weaklycurved classical supergravity. Moreover, the construction is explicitly holographic: the CFT lives on the boundary of $A d S_{5}$; each operator $\mathcal{O}$ of the CFT is identified with a bulk field in $A d S_{5} \times \mathbf{S}^{5}$ according to a standard "dictionary"; the sources for the CFT operators are taken to be the boundary values of the bulk fields; and the supergravity partition function $Z_{\mathrm{SG}}$ is identified as the generating functional of the CFT correlation functions. If one denotes the bulk field associated with an operator $\mathcal{O}$ as $\phi^{\mathcal{O}}$, then correlation functions in the CFT are given by

$$
Z_{\mathrm{SG}}\left[\phi_{0}^{\mathcal{O}}\right]=\int_{\phi^{\mathcal{O}} \rightarrow \phi_{0}^{\mathcal{O}}} D \phi^{\mathcal{O}} e^{-S_{S G}\left[\phi^{\mathcal{O}}\right]}=\left\langle e^{-\int_{\partial \mathrm{AdS}} \phi_{0}^{\mathcal{O}} \mathcal{O}}\right\rangle_{\mathrm{CFT}}
$$

The remarkable aspect of this construction is the strongweak duality. The regime where the gauge theory is strongly coupled corresponds to the weakly coupled 5D theory. Thus, the CFT correlation functions in the strongly-coupled regime are obtained from the supergravity action $S_{S G}$ evaluated with the classical solution for fields $\phi^{\mathcal{O}}$; these approach a specified boundary value $\phi_{0}^{\mathcal{O}}$, and appropriate functional derivatives with respect to $\phi_{0}^{\mathcal{O}}$ are taken. 23, 24].

The AdS/CFT correspondence shows that at least some gauge theories exist which have practical higherdimensional dual theories in the sense that they can be treated classically. Being gauge theories, they are relatives of QCD. The hope is that QCD can be treated similarly. Note that in constructing the practical dual theory for the CFT, two limits were critical: the large $N_{c}$ limit (which reduced the theory to SUGRA from a string theory) and the strong coupling limit (which renders the five-dimensional theory classical. Presumably some analog of these will be needed for holographic models of QCD. Thus one expects that a viable treatment of QCD will require the QCD to be in the large $N_{c}$ limit and the strongly coupled regimes. The large $N_{c}$ limit is straightforward intellectually - but has some important implications when attempts to do phenomenology. The strong coupling regime is more problematic and will be discussed below. 
The bottom-up approach to holographic QCD involves the following basic steps: guessing a 5D background; taking a field content that captures some aspects of large $N_{c}$ QCD for some observables of interest; a dictionary is used to relate the QCD operators to the bulk fields (this is generally taken to be the AdS/CFT dictionary using the naive dimensions of the QCD operators); guessing a form for the action of the five-dimensional theory; in building this action one typically uses the 5D masses obtained from the AdS/CFT dictionary. Once these steps are taken the problem becomes computational. This is called a "bottom-up" approach to distinguish it from a "top-down" philosophy in which one attempts to construct the dual theory from string theory rather than guess its form.

Some of the guesses in this game are easy to motivate. In AdS/CFT, the conformal invariance on the $4 \mathrm{D}$ side reflects coordinate rescaling as an isometry of AdS. Now there is a sense in which QCD is effectively conformal at high energies: due to asymptotic freedom at high momentum there is no scale remaining and things act conformally. However, at low momenta confinement sets a natural scale and effective conformal invariance is lost. Since confinement is critical to hadronic physics, an unmodified $A d S_{5}$ background can not capture the essence of QCD; minimally $A d S_{5}$ space must be modified to reflect confinement. Typically, the form guessed for the background in holographic QCD is asymptotically $A d S_{5}$ background near the UV-brane; this captured the effectively conformal nature of QCD at high energies. The deep bulk region - corresponding to IR physics - is then taken to deviate from AdS in order to model confinement. A popular simple choice is a hard wall [3, 4, , 5, , 7, 8, , 9, 12, 17], in which confinement is modeled by cutting off the AdS space at some finite radius by hand. The simplicity of such a model comes at phenomenological cost: the models do not produce a linear Regge meson mass spectrum as expected in QCD. Soft wall models cure this. They use a dilaton field in the bulk which is tuned to effectively cut off the AdS space in a smmoth way that is designed to reproduce the Regge spectrum [10, 17, 18, 19]. There are alternative approaches which models include the back-reaction of the bulk fields on the metric, which can dynamically cut off the AdS space [14, 15].

There are a number of issues which threaten the viability of such an approach. Perhaps the most obvious one is the reliance on a formalism taken over from AdS/CFT which depends on the strong coupling limit of the CFT. Now in a CFT, the coupling does not run and it is thus meaningful to ask whether the theory is strongly coupled. In QCD the coupling runs so it is difficult to even formulate the question of whether the theory is strongly coupled. To the extent the question can be posed it must be posed on a process-by-process basis. This is particularly worrying since at high momenta where QCD looks effectively conformal it is weakly coupled. Thus generically QCD, there is no region in which QCD is both very strongly coupled and effectively conformal. Accordingly it is highly questionable whether there is any region of QCD where it is legitimate to expropriate any aspects of the AdS/CFT formalism for this system.

There are a large number of subsidiary issues related to this. From the beginning many of the models fix parameters by matching to QCD in the UV [3, 4, 5]. In part this is done to limit the number of parameters so the model can have more predictive power and in part since QCD is tractable in this regime. However, this practice makes manifest the problem discussed above. It is based on matching to QCD in its weakly-coupling regime; yet the expectation is that the AdS/CFT construction which motivates the model is only valid for strong coupling.

Another conceptual issue with the implementation of the models concerns the AdS/CFT dictionary [23, 24] which is typically used without modification in the bottom-up approach. The bulk field content is determined by associating a p-form 4D QFT operator $\mathcal{O}$ with scaling dimension $\Delta$ to a p-form bulk field with the five$\mathrm{D}$ mass $m_{5}$ uniquely specified by $\Delta$ and $p$. The not so subtle issue here is that the operators in QCD run due to their anomalous dimensions - except for the case of conserved currents. This raises the obvious question of whether it is sensible to use the naive dimension of the operator in implementing the dictionary. As noted in ref. 31] this also raises phenomenological theoretical issues in fitting parameters associated with these operators unless the models can be somehow augmented to match the scale dependence known in QCD.

A related issue concerns the set of QCD operatorsand corresponding five-dimensional fields-included in the modeling. QCD has an infinite number of operators with any set of quantum numbers. In general these operators mix: the cross correlation functions are nonzero. Moreover there is no obvious suppression scale. Thus, in principle, an arbitrarily large subset of them can contribute to any given process 32]. An ad hoc approximation is typically made in order to construct tractable holographic models: the models typically are restricted to a minimal set of lowest-dimension operators which probe the quantum numbers of interest. Apart from its ad hoc nature this procedure raises a conceptual problem. Suppose that one assumes that in principle the models treated classically ought to be rich enough to reproduce any QCD correlation function, including the nonvanishing mixed correlators between distinct operators of the same quantum numbers. Suppose furthermore that one associates each of these operators with a distinct bulk field fixed by the AdS-CFT dictionary and use a classical treatment with some geometrical background and some action. Then in order to reproduce the QCD level correlators, it is apparent these distinct fields must interact classically (either in the bulk or on the brane). At this level there is no conceptual difficulty. However, now suppose we wish to obtain a simpler model with only a minimal set of fields (say one per quantum number); then one needs to integrate out the bulk field's extra degrees of freedom, yielding a generally nonlocal five-dimensional 
theory for the remaining degrees of freedom[10]. One might hope to make some type of field redefinition to yield an approximately local theory with the remaining fields. This poses a possible problem: one might expect that the modification of the action due to integrating out the extra fields and redefining the surviving ones can alter the $5 \mathrm{D}$ mass term in the action away from that given by the AdS/CFT dictionary. Thus it is unclear why the mass terms in the simple models are given by the dictionary.

There is another set of issues with bottom-up holographic models of QCD which needs to be addressed, namely, the extent to which models of this type are capable of reproducing the essential physics of various aspects of QCD. To make this question a bit more crisp we should recall that one of the key ingredients allowing for a simple classical treatment in the AdS/CFT formulation was the large $N_{c}$ limit. It is hard to believe that an AdS/QCD connection should be less dependent on large $N_{c}$. This does not mean that one cannot try to include some finite $N_{c}$ corrections when doing phenomenology. However, such corrections will presumably be included in an ad hoc way. The significant point here though is that to the extent a holographic model is viable for describing some class of phenomena, it should at least be able to describe the phenomena at large $N_{c}$. Since qualitatively much is known about large $N_{c}$ phenomenology there are important constraints on model building.

In fact, much of the modeling does depend critically on large $N_{c}$. For example, simple classical holographic models of mesons yield mesons with well-defined masses. To the extent widths are treated, they are included as perturbative corrections to a would-be stable state. In general such a treatment is not justified but it is at large $N_{c}$ where meson widths automatically go to zero as $1 / N_{c}[20,21]$. However, this does not apply to baryons which generically have a width of order $N_{c}^{0}$ if decays are kinematically allowed 21]. Thus holographic models of baryons such as those in [33] which use field operators taken to be dual to currents with baryon quantum numbers (in analogy to the treatment of mesons) and which give rise to excited baryons with well-defined masses appear to be inconsistent with large $N_{c}$ QCD. Moreover one cannot evade this problem by simply working at $N_{c}=3$ since baryons are not narrow in the $N_{c}=3$ world either. This difficulty need not exclude the possibility of describing baryons with holographic models [34] if one describes baryons arising essentially as Skyrmions[2], i.e, as solitons from the non-linear dynamics of the mesons. However, it does illustrate how the known large $N_{c}$ behavior constrains model building.

The question of whether a practical holographic model is capable of reproducing the properties of QCD matter at non-zero temperature is particularly important 35]. On the one hand, the description of the phase structure of strongly interacting matter and the problem of QCD at finite temperature more generally are at the core of modern nuclear physics and is tied to experimental studies of ultra-relativistic heavy ion collisions. However, there is a fundamental issue which arises in describing QCD at finite temperature using a practical holographic model. This is associated with a universal feature of holographic theories with five-dimensional supergravity duals. Consider a theory in the limit in which such theories may be treated classically on the $5 \mathrm{D}$ side (as is implicitly assumed to be true in practical holographic models of QCD). Furthermore assume that the $4 \mathrm{D}$ theory is in its deconfined phase. As noted by Witten such a phase is equivalent to there being a black hole in the geometry of the fivedimensional space 36. In such geometries all theories in the class under consideration have a ratio of shear viscosity to entropy density, $\eta / s$ given by $(4 \pi)^{-1}$ [37]. This presents a fundamental difficulty in trying to describe QCD at high temperatures. We know that as $T$ gets large, $\eta / s$ should diverge in a logarithmic manner with $T$ due to asymptotic freedom; practical holographic models based on SUGRA cannot describe this regime. One might try to evade this problem by adding higher-order terms to the gravity theory which allows $\eta / s$ to differ from $(4 \pi)^{-1}$ [38]. However it is by no means clear that there is a viable way to do this. An alternative strategy is to suggest that the models are only valid for describing finite temperature QCD up to a value not too far above the critical temperature $T_{c}$ [35]. For this strategy to be viable, $\eta / s$ at $T_{c}$ in large $N_{c}$ QCD needs to be $(4 \pi)^{-1}$ or very close. A priori there is no reason to believe that this is true. The phase transition is first order at large $N_{c}$ meaning it is driven by global energetics rather than local properties of the phase. Thus nothing special happens in the deconfined phase as one approaches $T_{c}$ from above. Accordingly it is very hard to conceive of any reason why one would expect $\eta / s$ to approach $(4 \pi)^{-1}$ as $T$ approaches $T_{c}$.

The crux of this problem is the one discussed earlier: QCD has both strongly coupled and weakly coupled aspects. The high temperature phase of QCD has weakly coupled quarks and glue and this is precisely what causes $\eta / s$ to diverge. On the other hand, the classical treatment of the 5D models is only expected to work for strongly coupled theories. Thus at high temperature there is a fundamental tension between the dynamics of QCD and the structure of practical holographic models.

There is a final set of challenges to consider. As noted earlier, at best the practical holographic models of QCD are justified in the large $N_{c}$ limit. To the extent that some $1 / N_{c}$ corrections are included (by, for example, fitting parameters to real world data) they are not included in any systematic way. The large $N_{c}$ limit and the $1 / N_{c}$ expansion are of use in doing phenomenology only to the extent that the large $N_{c}$ world is at least a rough caricature of the $N_{c}=3$ world. However, the extent to which the large $N_{c}$ world resembles the physical world is highly dependent on which observable one is interested in studying. It is often the case for hadronic observables that the large $N_{c}$ limit does provide a recognizable, if crude, of the physical world. However, even in the domain of 
hadronic physics there are cases where the standard large $N_{c}$ treatment fails phenomenologically. The most wellknown example of this concerns the $U(1)$ problem 39 . Thus, one should view results associated with the $\eta^{\prime}$ meson in holographic models with particular caution.

More importantly as one goes away from the domain of hadronic physics to issues in nuclear physics as in ref. [41], the large $N_{c}$ limit and, hence, practical holographic models becomes more problematic. The difficulty has to do with energy and momenta scales in nuclear physics which are characteristically much smaller than in hadron physics. Characteristic energies and momenta in hadronic physics are typically hundreds of $\mathrm{MeV}$ to a $\mathrm{GeV}$. So long as the energetics associated $1 / N_{c}$ effects are small on this scale it is presumably legitimate to use a $1 / N_{c}$ expansion and truncate it at relatively low order. A critical energy scale to keep in mind is the nucleon- $\Delta$ mass splitting, a $1 / N_{c}$ effect which is numerically $\sim 290$ $\mathrm{MeV}$. The $1 / N_{c}$ expansion for baryons depends on the excitation of typical baryons to be large compared to this. In practice this is somewhat marginal for many baryons and the $1 / N_{c}$ expansion at low order for such cases typically should be considered to be semi-quantitative. However, in the domain of nuclear physics the characteristic energy scales are much smaller. For examples the binding energy of the deuteron is approximately $2 \mathrm{MeV}$ or the binding energy per particle of nuclear matter is approximately $16 \mathrm{MeV}$. Using Witten's standard analysis[21], it is easy to see that nuclear binding energies are of order $N_{c}^{1}$ - just as baryon masses are. Thus, order $N_{c}^{1}$ nuclear quantities such as these binding energies are smaller by more than an order of magnitude than the nucleon- $\Delta$ splitting which is formally of order $1 / N_{c}$. This strongly suggests that treatments based on the leading order of the $1 / N_{c}$, such as classical treatments of the Skyrme model or holographic models of QCD, are likely to fail to describe nuclei accurately. Configurations which admix $\Delta$ components can be included with no energetic penalty due to the N- $\Delta$ mass difference in such treatments. Such admixtures will be induced to maximize attraction. However, repulsive contributions due the N- $\Delta$ which are formally higher order in $1 / N_{c}$ are likely to be much larger than the physical binding energies given the small size of the latter compared to the former.

The preceding argument strongly suggests that while a treatment of low energy nuclear structure based on the leading order $1 / N_{c}$ expansion might be valid for, say, a world with $N_{c}=1001$, it is almost certainly not valid for the real world of $N_{c}=3$. This in turn implies that whatever else practical holographic models of QCD may be good for, they are very unlikely to be useful phenomenological models for nuclear structure. Of course, holographic models are not special in this regard; any model of the strong interactions which relies on the large $N_{c}$ limit is unlikely to be capable of describing nuclear structure.

There is another important domain in which reliance on the large $N_{c}$ limit implicit in practical holographic models of QCD is likely to be problematic phenomenologically: the finite temperature domain near the QCD phase transition. It is believed that the QCD phase transition at large $N_{c}$ is strongly first order with a latent heat of order $N_{c}^{2}[21]$; this is consistent with what has been observed on the lattice [40]. On the other hand, QCD with $N_{c}=3$ and two light but not massless quarks, there is no phase transition at all but a crossover; as the quark masses go to zero a second-order phase transition occurs. Thus the transition region in the physical world and the extrapolation of the physical world to the chiral limit looks qualitatively quite different than the large $N_{c}$ limit. This is a problem because any attempt to describe this physics in a practical holographic model will necessarily depend on uncontrolled higher-order corrections in a $1 / N_{c}$ expansion. An optimistic view is that the large $N_{c}$ limit could still accurately describe the system except in a narrow region around the phase transition. However, it is worth noting from the discussion of $\eta / s$ that the models are expected to fail well above $T_{c}$ and from the discussion above it seems apparent that they also are expected to fail near $T_{c}$. This raises an obvious question: Is there any region in the deconfined phase where the models do work?

In summary, practical "bottom-up" holographic models of QCD are quite interesting. However, they face a number of challenges both at the theoretical and phenomenological levels. Before accepting models of this class as useful descriptions of strong interacting physics it is important that these be addressed. It also seems clear that at best the domain of validity of these models is likely to be rather limited.

\section{Acknowledgments}

The support of the United States Department of Energy is gratefully acknowledged. Discussions with A. Cherman were extremely helpful in formulating the arguments in this paper.
[1] M.C. Birse, M.K. Banerjee Phys. Lett. B136 (1984) 284; Phys. Rev. D31 (1985) 118.

[2] T.H.R. Skyrme Proc. Roy. Soc. Lond. A260 (1961) 127; Nucl. Phys. 31 (1962) 556; for a review of the Skyrme model see I. Zahed and G.E. Brown, Phys. Rept. 142
(1986) 1.

[3] J. Erlich, E. Katz, D. T. Son and M. A. Stephanov, Phys. Rev. Lett. 95, 261602 (2005) arXiv:hep-ph/0501128.

[4] L. Da Rold and A. Pomarol, Nucl. Phys. B 721, 79 (2005) arXiv:hep-ph/0501218. 
[5] L. Da Rold and A. Pomarol, JHEP 0601, 157 (2006) arXiv:hep-ph/0510268.

[6] K. Ghoroku, N. Maru, M. Tachibana and M. Yahiro, Phys. Lett. B 633, 602 (2006) arXiv:hep-ph/0510334.

[7] E. Katz, A. Lewandowski and M. D. Schwartz, Phys. Rev. D 74, 086004 (2006) arXiv:hep-ph/0510388

[8] J. Hirn, N. Rius and V. Sanz, Phys. Rev. D 73, 085005 (2006) arXiv:hep-ph/0512240.

[9] J. Erlich, G. D. Kribs and I. Low, Phys. Rev. D 73, 096001 (2006) arXiv:hep-th/0602110.

[10] A. Karch, E. Katz, D. T. Son and M. A. Stephanov, Phys. Rev. D 74, 015005 (2006) arXiv:hep-ph/0602229.

[11] S. J. Brodsky and G. F. de Teramond, Phys. Rev. Lett. 96, 201601 (2006) arXiv:hep-ph/0602252.

[12] J. P. Shock and F. Wu, JHEP 0608, 023 (2006) arXiv:hep-ph/0603142

[13] N. Evans, A. Tedder and T. Waterson, JHEP 0701, 058 (2007) arXiv:hep-ph/0603249

[14] C. Csaki and M. Reece, JHEP 0705, 062 (2007) arXiv:hep-ph/0608266.

[15] J. P. Shock, F. Wu, Y. L. Wu and Z. F. Xie, JHEP 0703, 064 (2007) arXiv:hep-ph/0611227.

[16] T. Schafer, arXiv:0711.0236 [hep-ph].

[17] H. Forkel, arXiv:0711.1179 [hep-ph].

[18] H. J. Kwee and R. F. Lebed, arXiv:0712.1811 [hep-ph]

[19] P. Colangelo, F. De Fazio, F. Jugeau and S. Nicotri, arXiv:0711.4747 [hep-ph]

[20] G. 't Hooft, Nucl. Phys. B 72, 461 (1974).

[21] E. Witten, Nucl. Phys. B 160, 57 (1979).

[22] J. M. Maldacena, Adv. Theor. Math. Phys. 2, 231 (1998) [Int. J. Theor. Phys. 38, 1113 (1999)] arXiv:hep-th/9711200

[23] S. S. Gubser, I. R. Klebanov and A. M. Polyakov, Phys. Lett. B 428, 105 (1998) arXiv:hep-th/9802109

[24] E. Witten, Adv. Theor. Math. Phys. 2, 253 (1998) arXiv:hep-th/9802150.

[32] Y. A. Glozman, Private communication, 2007.

[26] For a review, see K. Skenderis, Class. Quant. Grav. 19,
5849 (2002) arXiv:hep-th/0209067

[27] I. R. Klebanov and E. Witten, Nucl. Phys. B 556, 89 (1999)

[28] E. Shuryak, arXiv:0711.0004 [hep-ph].

[29] N. Evans, J. P. Shock and T. Waterson, Phys. Lett. B 622, 165 (2005).

[30] N. Evans and A. Tedder, Phys. Lett. B 642, 546 (2006) arXiv:hep-ph/0609112.

[31] A. Cherman, T.D. Cohen and E.S. Werbos, arXiv:0804.1096

[32] L.Ya. Glozman, private communication.

[33] arXiv: G.F. de Teramond and S.J. Brodsky hep-th/0409074 Phys. Rev. Lett. 94 (2005) 201601.

[34] K. Nawa, H. Suganuma and T. Kojo hep-th/0612187 H. Hata, T. Sakai, S. Sugimoto, S. Yamato hep-th/0701280 D.K. Hong, M. Rho, H.-U. Yee, P. Yi arXiv:0705.2632

[35] K. Ghoroku and M. Yahiro Phys. Rev. D73 (2006) 125010; K Ghoroku, A. Nakamura and M. Yahiro Phys. Lett. B638 (2006) 382; C.P. Herzog Phys. Rev. Lett. 98 (2007) 091601; K. Kajantie, T. Tahkokallio, J.T. Yee JHEP 0701 (2007) 019;Oleg Andreev, Phys. Rev. D76 (2007) 087702; U. Gursoy , E. Kiritsis, L. Mazzanti,and F. Nitti arXiv:0804.0899, S.S. Gubser, A. Nellore arXiv:0804.0434, S.S. Gubser, A. Nellore, S.S. Pufu, F.D. Rocha arXiv:0804.1950

[36] E. Witten Adv. Theor. Math. Phys. 2 (1998)505.

[37] A. Buchel and J.T. Liu, Phys. Rev. Lett. 93 (2004) 090602; P. Kovtun, D.T. Son and A.O. Starinets, Phys. Rev. Lett. 94 (2005) 111601.

[38] M. Brigante, H. Liu, R.C. Myers, S. Shenker and S. Yaida, arXiv:0712.0805

[39] E. Witten Nucl. Phys. B156 (1979) 269; G. Veneziano Nucl. Phys. B159 (1979) 213.

[40] B. Lucini, M. Teper and U. Wenger JHEP 0502 (2005) 033.

[41] Y. Seo and S.-J. Sin arXiv:0802.0568, Y. Kim, C.-H. Lee and H.-U. Yee arXiv:0707.2637 